\title{
A Qualitative Study on the Feasibility and Acceptability of Establishing Health Technology Assessment (HTA) in a Low Income Country Setting: The Case of Malawi
}

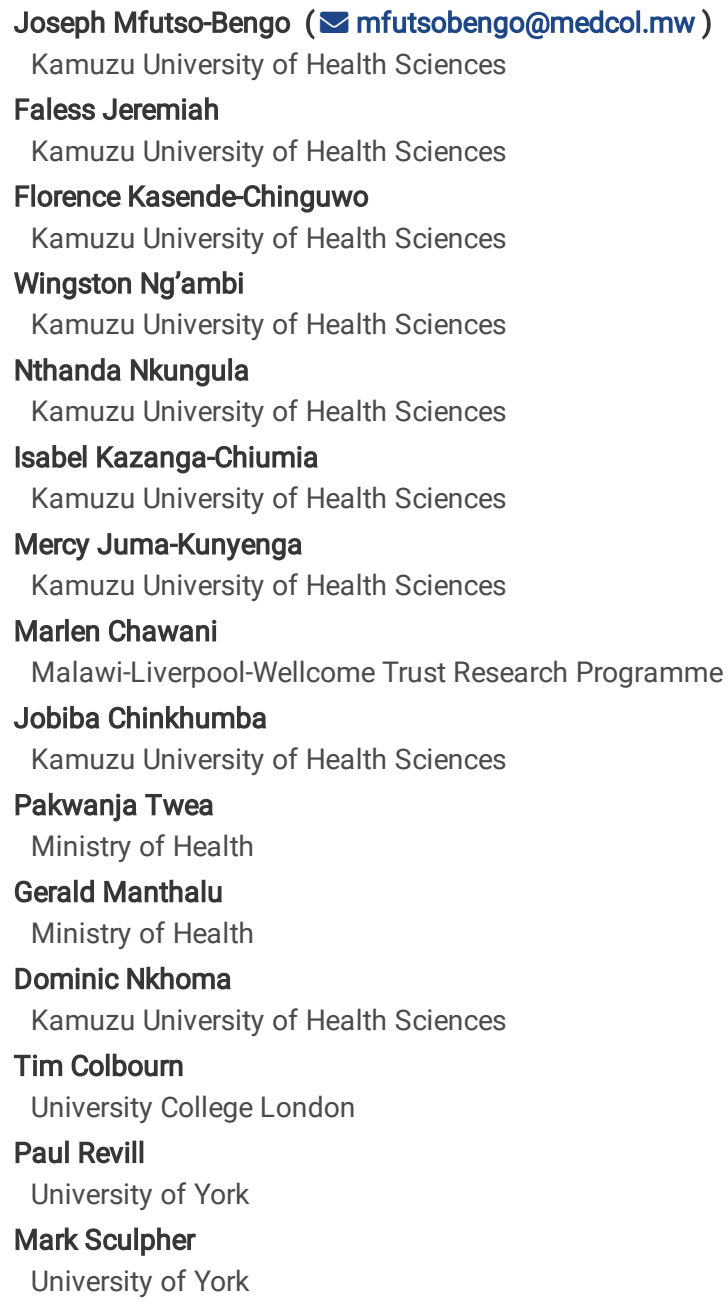




\section{Abstract}

Background

The objective of this study was to assess the feasibility and acceptability of institutionalizing Health Technology Assessment (HTA) in Malawi focusing on the form, scope, capacity and financing requirements for a limited resource setting such as Malawi.

Methods

This study employed a desk review and qualitative research methods. An extensive literature review was conducted on relevant key policy documents, reports and publications to understand the status of HTA in Malawi. The study included one focus group discussion with 7 participants and 12 key informant interviews with key stakeholders selected purposely based on their knowledge and expertise in HTA.

Results

The study found that partial or informal HTA processes exist and that HTA is practiced without a standardized framework. The partial HTA processes are executed through three structures; Ministry of Health Senior Management Team, Technical Working Groups and Pharmacy and Medicines Regulatory Authority (PMRA) with varying levels of effectiveness. The results revealed that lack of an institutional HTA creates severe shortfalls. This includes partial HTA mechanisms, limited evidence use, especially concerning cost-effectiveness, lack of a standardized framework for technology adoption, donor pressure, lack of resources for the HTA process and technology acquisition, laws and practices that undermine cost-effectiveness considerations.

Conclusion

Institutionalizing HTA in Malawi is acceptable and feasible. The results suggest that any HTA institutionalization in the short to medium term could involve strengthening the capacity of PMRA to appraise Cost-effectiveness Analysis (CEAs) within academia and MOH health financing division. Furthermore, rather than creating new HTA institutions, country-specific assessments should precede any specific recommendations for new technology adoptions. A technical capacity in Academic institutions such as Health Economics and Policy Unit (HEPU) at Kamuzu University of Health Sciences (KuHes) can be developed either as "offshore" HTA capacity fully aligned to $\mathrm{MOH}$ and executing HTA evidence and knowledge management function in a sub-contractor capacity.

Running Head

Health technology assessment in Malawi

\section{Introduction}

Health systems in low-income countries (LICs) such as Malawi are characterized by low per capita health spending. Limited budgets for effective health care provision have created a dire need for stronger resource allocation and prioritization processes as opportunity costs in sub-optimal decision making lead to significant losses in the populations' overall health gains (1). Evidence internationally proffers that LICs face systematic challenges in optimizing already scarce resources in their health sectors. These challenges therefore undermine service delivery and the provision of equitable and quality health care that could easily be remedied through increased and efficiently allocated healthcare finances (2). Thus, the pursuit of a strategy of evidence-based and optimal value based decision-making frameworks through tools such as Health Technology Assessment (HTA) can help address issues relating to both affordability and equity in priority setting when allocating extremely scarce resources in these contexts (3).

A health technology is defined as a health intervention that seeks to improve health and health sector outcomes by "preventing, diagnosing and treating medical conditions, promoting health or organizing health delivery" (4). HTA is subsequently defined as "the systematic evaluation of the properties and effects of a health technology aimed at addressing the direct and intended effects of that health technology, as well as its indirect and unintended consequences" (4). HTA is largely propagated in areas that target efficient and cost effective health resource allocation, and in the prioritization, application and adoption of varied health service technologies (4). As a result, the widespread use of HTA holds prominence in medical, organizational, economic, ethical and societal means for technology espousal for a nations health sector at all levels of development (5).

Globally, institutionalized HTA is mostly used in the context of high- and middle-income countries like the United Kingdom famous NICE structure, Australia, Thailand and several European countries. In these contexts, HTA has a precarious role in prioritization and decision-making. Further, HTA in these contexts brings forth clear and standardized decision-making institutions that provide assessment mechanisms that contribute in cost-effectiveness analyses and evidence provision. All these lead to quality health care intervention adoption for their respective populations (2). On the other hand, decision-making structures in many LICs like Malawi lack institutionalization, resulting in the inefficient and cost - ineffective allocation of scarce health care (3).

This study sought to assess the feasibility and acceptability of implementing an institutionalized HTA mechanism for the Malawian healthcare sector. In particular the study focused on assessing the; (a) current value sets that aid decision making practices in health technology adoption in Malawi, (b) current decision making framework for health technology adoption in Malawi, (c) determine the funding structure for health technology assessment and adoption, and (d) willingness of policy makers and relevant stakeholders to adopt an institutionalized HTA structure for Malawi and the (e) viability of the Malawian context to accommodate and institutionalize HTA (feasibility). 
"Institutionalizing" refers to the establishment of governance structures and pathways appropriate to yield technology assessments that are fundamental in guiding policy and clinical practice towards the best possible health and cost outcomes (6). The WHO endorsed HTA as a tool to support open, "evidence-informed priority setting, through systematic evaluation of the properties and effects of a health technology" (7). The use of HTA in LMICs is further advocated to determine the value of a health technology (e.g., a drug, medical device, diagnostic test, medical procedure) at different points in its lifecycle with an aim of informing the decision making to promote an efficient, equitable, and high-quality health system (8).

For a country to effectively achieve priority setting it needs an institutionalized HTA. The tenets behind the successful institutionalizing of HTA as advocated by WHO are establishing the demand for HTA; establishing institutional arrangements; establishing a legal framework, Human resources capacity for HTA, HTA financing, HTA evidence and evaluation of the HTA mechanism (5). Institutionalizing HTA promotes structures and processes suitable to produce technology assessments that are optimal in guiding policy and clinical practice towards the best health and cost outcomes (8). The aim is to use HTA among relevant stakeholders to address health sector allocation challenges closing the knowledge gaps among HTA stakeholders and users directed towards improved and efficient service delivery outcomes (9). Diagram 1 depicts the conceptual framework for institutionalizing HTA in a healthcare system, were the premise lies; HTA dimensions of agenda setting, having appropriate values for decision making, including robust evidence processes and institutional arrangements will thus lead to efficient and effective use of health care resources thus contributing towards the goal of UHC through efficient access to health resources (10). There are no standardized models or universal paths for the development and institutionalization of agencies but rather considerations maybe established on the basis of a countries cultures and values, healthcare systems, political priorities and governance (11).

\section{Methods}

This assessment employed desk review and purposefully curated qualitative research methods. An extensive literature review was conducted on relevant key policy documents, reports and publications on HTA in Malawi. Key relevant documents were identified through consultation with key informants from the Ministry of Health and online literature search. PubMed, Cochrane, BMC and Science Direct were among the databases searched. Key terms such as "health technology assessment", "HTA values", "structure of HTA in (country)", "Southern African HTA Association" were used to identify relevant articles. The search was limited to articles published after the year 2010 and written in English and excluded articles whose emphasis was not in the HTA tool. The aim of the literature review was to benchmark HTA institutions or structures in various countries drawing lessons on to how HTA could be institutionalized in Malawi. We also consulted the websites of INAHTA; Health Technology Assessment International (HTAi); EUnetHTA; and those of other relevant HTA. National Institute for Clinical Excellence (NICE), United Kingdom; Agency for Healthcare Research and Quality (AHRQ), United States; Institute for Quality and Efficiency in Health Care (IQWiG), Mexico; Haute Autorité de Santé (HAS), France; National Committee for Health Technology Incorporation (CONITEC), Brazil; and the Institute of Health Technology Assessment (IETS), Colombia. These organizations were selected to further the literature-review based analysis.

The qualitative study employed one Focus Group Discussion (FGD) with 7 policy makers and 12 Key Informant Interviews (KIIs) with key stakeholders. The whole qualitative sample constituted of 19 participants employed based on data saturation. The FGD and the KII were conducted with interview guides informed by the literature and the KII were done iteratively to ensure that adequate data was collected for the research question. The participants included officials from the Ministry of Health (Headquarters, Central Hospitals, District Hospitals), civil society, Central Medical Stores Trust (CMST), Christian Health Association of Malawi (CHAM) and regulatory authorities i.e. Medical Council and Pharmacy and Medicines Regulatory Authority (PMRA). The FGD was used to gain understanding of the decision-making processes related to health technology adoption in Malawi whilst the KII's were used to gauge further individual perceptions, beliefs, and attitudes on identifying, prioritization, adopting and implementation of health technologies in Malawi.

The data was collected between November 2020 and March, 2021. Due to COVID-19 restrictions, interviews and the FGD were conducted primarily via phone call and Zoom with the exception of four face-to-face interviews that adhered to COVID - 19 guidelines. All interviews and the discussions were audio-recorded with unique identification assigned to each recorded interview. To enhance credibility, field notes were captured during data collection as well as triangulation discussions among researchers. All interviews were conducted in English as per participants' preference. The audio data were transcribed verbatim into Microsoft Word with data analysis conducted using thematic analysis approach. Systematic coding was done and a code book in excel was used to organize and manage the data. Two researchers independently coded separate transcripts to assess the applicability of codes and any differences were resolved through iterative discussions between them. These were then validated by a third researcher. The themes were developed and refined by organization into extracts of coherent patterns and meaning was ascertained in coherence to how they related to the relevant research questions. The broad questions which guided the KII and FGD were as follows:

1. What is the process for introducing medicines (pharmaceuticals), diagnostics/medical equipment and vaccines?

2. Who are the main actors in the decision-making processes for identification, prioritization and adoption of health technologies in Malawi?

3. What value sets and frameworks are used to guide the identification, prioritization and adoption of health technologies?

\section{Ethical Consideration}

The study observed strict ethical considerations approved by the College of Medicine Research Committee (COMREC) in Blantyre, Malawi (protocol \#: P.10/19/2820). All methods were performed in accordance with the relevant guidelines and regulations (declaration of Helsinki). Verbal consent was obtained from all the participants involved. Participants who took part in the FGD were requested to observe confidentiality. To protect the confidentiality of the participants' information; no identifying information was included on the interview transcripts or FGD. The audios and transcripts were kept in a password protected computer with access limited to the researchers. 


\section{Results}

This section presents findings of the desk review and qualitative study on the feasibility and acceptability of institutionalizing HTA in Malawi commencing with the current decision making processes that guide prioritization and adoption of health technologies in Malawi. The study further goes on to illustrate mechanisms of how HTA is being conducted in various contexts across the world.

\section{Current Decision Making Structures for Health technology adoption in Malawi}

The participants from the FGD and KIIs indicated that Health Technologies in Malawi include pharmaceuticals/medicines, medical equipment and vaccines. Apart from the policymakers at the ministry of health, there are other players in adopting new health technologies (for instance, PMRA, Civil society, Malawi Medical council, non-governmental health care providers such as CHAM). The FGD participants indicated that health technology adoption in Malawi is primarily reliant on the funding source for corresponding health technology. The funding source may be in the form of: (a) government resources, (b) development partner funds, (c) donations in kind and (d) private sector funds. The funding source is key in determining adoption or nonadoption of each technology. We examined the current informal HTA mechanisms that exist.

\section{Government-funded technologies}

The findings from the FGD and KIls indicate that the decision-making process for health technology adoption in the healthcare system in Malawi is embedded within the Ministry of Health Senior Management Team (SMT) decision-making structure. The SMT comprises the Secretary for Health, Chief of Health Services, Directors and Deputy Directors of MOH Headquarters and Directors of the five Central Hospitals. Analysis of both KII and FGD, as well in conference with the desk review results, indicate a three-stage process. First, a relevant department identifies a health technology it needs and its director presents details of the health technology of interest to a relevant Technical Working Group (TWG). A TWG membership comprises technicians from the $\mathrm{MoH}$ and stakeholders with appropriate expertise. When more information is needed, it is not uncommon for a TWG to appoint a taskforce to undertake a more detailed analysis and present its findings for review and endorsement by the TWG. Following the review of the results, a TWG can recommend to the SMT of the $\mathrm{MOH}$ to adopt or not adopt the proposed health technology intervention. Based on the recommendation from the TWG, MoH SMT have the liberty to agree with and adopt the recommendation of the TWG, reject it altogether, or refer the work back to the TWG to address any areas that SMT advises on.

The qualitative inquiry further found that to implement the adoption decision from SMT, the user department initiates a procurement request which goes through the Internal Procurement and Disposal Committee (IPDC) for scrutiny. The main task of the IPC is to ensure that the acquisition of the technology complies with the Public Procurement Act of 2017. For technologies where the regular membership of the IPDC lacks some competencies, the committee can co-opt non-regular technical experts to complement its capacity. Based on feedback from the FGD, some of the key considerations that technical experts prioritize include "the functionality of the product or the equipment or the service in country, availability of capital sale services and financial capacity of the bidder... [...]; if given a contract [they] should be able to bring the product into the country" (FGD Res.07_Policy Maker).

For these Government funded technologies, the implementation of the decision by SMT is generally executed by the Department of Health Technical Support Services ( ${ }^{[1]}$ DHTSS) but there are others such as the Diagnostics Unit, Clinical department and the Public health institute. The DHTSS has three Divisions: 1) the Physical Asset Management (PAM) Unit coordinates procurements of technologies that focus on medical devices, 2) the Pharmaceuticals Division, which coordinates the procurement of medicines and medical supplies; and 3) the Laboratory Unit which coordinates procurement of laboratory supplies and reagents. The Pharmacy and Medicines Regulatory Authority (PMRA) provides regulatory oversight over medicines. However, its mandate has recently been expanded to cover diagnostics and monitor the safety and efficacy of the medicines and enforce compliance to standards. A respondent for instance indicated that "... when we see that the government would like to deal with that supplier, we write them that can you submit the dossiers, and an application to us so that we (can) assess your product" (KII 10_Regulatory Authority).

\section{Development partner funded technologies}

For development of partner-funded technologies, the government generally expresses the need for a technology to the donor through the coordinating department or program head. Mostly, requests for technology acquisition would be based on the priorities in the Health Sector Strategic Plan (HSSP) or a relevant program strategic plan such as the National Strategic Plan for HIV/AIDS. Two possibilities exist. The first is where the partner could provide the funds to the $\mathrm{MOH}$, for example, through the Health Services Joint Fund (HSJF) (a pooled fund for three Donors: UK Foreign, Commonwealth \& Development Office, Norway and Germany), or via a Project Implementation Unit (PIU) such as the joint PIU for GAVI and the Global Fund. In this case, the government-funded technology mechanism described above is utilized with or without adaptation depending on additional donor procedures for acquiring technologies. Other donor funded projects such as the HSJF use a Fiscal Agent who reviews requests approved by SMT to evaluate any potential fiduciary risk before acquiring the technology. The second is where donor-funded projects use their internal procurement systems to acquire the technology on behalf of the Government. In the second option, donor procedures will be used only after the technology has been approved by the regulatory body for the technology as explained by one FGD participant: "But in cases of emergency, sometimes it goes directly from the head of department to a partner such as CDC, CHAI, or UNICEF to help with procurement. In which case, the procurement process happens in their system now, not the government system. And once a technology has been procured, it's delivered to the government [which] facilitates distribution". (FGD Res 2_Policy maker)

\section{Technologies donated in kind}


There are two types of donations, fully funded donations and partially funded donations. For Fully funded donations, the total cost of acquiring the health technologies is fully covered by the donor. The need for such technologies is ideally expressed by the MOH through the relevant head of department or program However, it is not uncommon for technologies that are not deemed priorities for the Government to be donated. For donations that are made in direct response to an expressed need from the Government, the responsible head of department or program institutes a task force to provide advice on the safety, efficacy and appropriateness of the technology. The Malawi Bureau of Standards and World Health Organization standards are mostly used to inform this assessment. The taskforce also assesses the alignment of the health technology with national health priorities and feasibility of implementation. Based on the recommendation from the taskforce, a report is written to the Secretary for Health for review and possible approval that the donation be accepted. For partially donor funded technologies (donations in-kind), "at the point of acceptance the ministry should have appropriated the resources to support the activities that are being financed from the government side. (FGD Res 06). Thus, given that some obligation falls on the Government, the adoption process of the technology will proceed as under government funded technologies. The participants also reported that for donations that are not priority for $\mathrm{MoH}$, there is no distinct "HTA"

\section{Health technologies funded and distributed by the private sector}

Based on the PMRA Act of 2019, for pharmaceuticals that are procured and distributed by private entities, the PMRA is responsible for their review and registration. The scope of the PMRA has recently been expanded to regulate Allied Substances (Acaricides, Cosmetics, disinfectants, food Supplements, feed additives and supplements, traditional medicines), medical and surgical sundries, medical devices, reagents and condoms in addition to medicines. As a result, the PMRA undertakes technological assessments across pharmaceuticals and medical diagnostics.

To register pharmaceuticals, a company applies to PMRA expressing intent to sell products on the Malawian market. PMRA then inspects the company to check its compliance with WHO Good Manufacturing Practice as required by the law and issues a compliance certificate if standards are met. The registration process involves several steps including: application for registration of the product; undertaking a bioequivalent study or laboratory proxy; presentation of assessment report to the medicines committee of the PMRA Board. If endorsed by the committee, the report is then submitted to the PMRA board for approval after which the product is registered and adopted in Malawi.

“... there are product assessment procedures, clinical trial reviews, and pharmacovigilance safety surveillance of medicines... the (PMRA) team makes a report on every product found in retail pharmacies. They produce a report and submit it to the medicines committee of the board to consider the findings of the report... This committee escalates this process to the level of the board. Now the board ratifies that these products... have now been registered, they can be found on the Malawi market." (KII 10)

\section{Developing and determining essential medicines and equipment lists covered}

The process of developing essential medicines list involves the Malawi National Medicines Committee developing Malawi standard treatment guidelines which in turn incorporates the essential medicines list. The Malawi National Medicines Committee gets feedback from health care workers in various facilities who recommend which medicines to include or exclude from the list depending on their experiences as well as the prevailing disease burden in the country. However, the study participants felt that this decision making process need to be improved so that it uses more evidence than just health care worker's experiences.

\section{HTA Mechanisms that exist in Current Decision-Making Structures}

Based on funding streams, three distinct decision-making structures were identified, and these structures were found to execute certain but not all aspects of an HTA process. The informal HTA mechanisms were identified within the MOH Senior Management Team, Technical Working Groups, and the PMRA processes. The decision pathway has been mapped out in Table 1 below.

Table 1: HTA functions performed by MOH SMT, TWGs, and the PMRA 


\begin{tabular}{|c|c|c|c|}
\hline \multirow[t]{2}{*}{ HTA Process } & \multicolumn{3}{|l|}{ HTA Functions Performed } \\
\hline & MOH Senior Management Committee & Technical Working Groups & PMRA \\
\hline $\begin{array}{l}\text { Identification } \\
\text { and } \\
\text { prioritization }\end{array}$ & $\begin{array}{l}\text {-Determining technologies valuable to } \\
\text { the country and requiring HTA } \\
\text { - }\end{array}$ & $\begin{array}{l}\text {-Advising SMT and donors on } \\
\text { technologies requiring HTA }\end{array}$ & $\begin{array}{l}\text {-Conducting pharmacovigilance studies to } \\
\text { inform decisions for adopted technologies } \\
\text { - Health technology assessment done on } \\
\text { reactive basis }\end{array}$ \\
\hline Evidence & $\begin{array}{l}\text { - Not used consistently relies more } \\
\text { on expertise of members }\end{array}$ & $\begin{array}{l}\text {-inconsistent use of evidence } \\
\text {-CEA use in EHP revision that has } \\
\text { been operational over time }\end{array}$ & $\begin{array}{l}\text { - reasonable evidence use identified } \\
\text { - evidence synthesis becomes a challenge }\end{array}$ \\
\hline \multirow[t]{5}{*}{ Adoption } & $\begin{array}{l}\text {-Decision on what interventions to } \\
\text { include or exclude in essential } \\
\text { medicines list }\end{array}$ & $\begin{array}{l}\text {-Advice on what interventions to } \\
\text { include or exclude in essential } \\
\text { medicines list }\end{array}$ & $\begin{array}{l}\text {-(De) Registering of technologies } \\
\text {-Regulating clinical trials }\end{array}$ \\
\hline & -Decision on whether to fund or not & -Evidence synthesis & \\
\hline & $\begin{array}{l}\text {-Resource allocation and funding of } \\
\text { approved technologies }\end{array}$ & -Economic Evaluation & \\
\hline & $\begin{array}{l}\text {-Reviewing and determining the } \\
\text { essential health package for a given } \\
\text { period }\end{array}$ & & \\
\hline & $\begin{array}{l}\text {-Approval of donor funded and donated } \\
\text { technologies }\end{array}$ & & \\
\hline \multirow{2}{*}{$\begin{array}{l}\text { Implementation } \\
\text { and scale-up }\end{array}$} & -Decision on whether to scale up or not & -Advice on technologies to scale up & -Regulation of technologies \\
\hline & -Funding of technologies & & -Pharmacovigilance studies \\
\hline $\begin{array}{l}\text { Further } \\
\text { research } \\
\text { initiatives }\end{array}$ & - & $\begin{array}{l}\text {-Appraising evidence for } \mathrm{MOH} \text { and } \\
\text { donor decision making }\end{array}$ & -Conducting clinical trials \\
\hline
\end{tabular}

Based on the above analysis we can extract the decision process flow in diagram 2 below.

The PMRA Act presently mandates institutions to undertake some HTA processes for diagnostics but based on the interview results, this is not yet institutionalized. Specifically, the interview results showed that for all pharmaceuticals, the requirement to have them registered before they are prescribed directs some of the HTA process through PMRA. For medical diagnostics, there was no evidence for a dedicated institution and some HTA processes are undertaken through the TWG and any specifically instituted taskforce via the user department.

\section{Values and Principles Guiding HTA Practices in Malawi}

Technology adoption for Government and donor funded technologies is guided by existing national priorities in key policy and strategic documents. These policies and guidelines include the recently revised Essential Health Package (EHP) (19), National Health Policy, Health Service Strategic Plan II (HSSP II) (20), Essential Medicines List (21), Standard Treatment Guidelines (21), Laboratory policy (22), Procurement Act (23) and WHO guidelines. Other normative values that were mentioned by some include appropriateness, affordability, accessibility, maintenance cost and functionality of the products. An assessment of the policy documents revealed that these values are included as guiding principles without interventions and indicators for ensuring the relevant organizations build the relevant culture in their decision-making, including HTA.

The key observation provided on the use of values and principles is that they are not always adhered to leading to the misalignment of normative and actual values that are practiced as one respondent from the FGD reported. This example was given in light of the recently revised Essential Health Package (EHP) revision, were it being the mother policy was revised but its corresponding policies such as the Essential Medicines List and the Standard Treatment Guidelines were not revised leading to a misalignment or in congruency in policy implementation. "....although there are value sets which define the EHP but the process of letting that process inform say the treatment guidelines or essential medicines list that process isn't in existence now. So it's one way the values are there but am no sure that they are being translated into the process that really informal what's procured." (FGD Res.005).

\section{Strengths and challenges of partial HTA mechanisms in current decision-making structures}

To determine whether institutionalizing HTA is feasible, it is important to understand the strengths, weaknesses, opportunities and threats posed within each of the informal HTA mechanisms that have been identified within the decision-making structures. The respondents reported various challenges affecting the current decision making processes of assessing health technologies for adoption in the Malawian health system. These include limited use of evidence, lack of a standardized framework, political interference, lack of resources, pressure from donors, difficulties in implementing cost-effective interventions due to conflicting policies, lack of transparency and accountability.

Limited use of Evidence: Despite that evidence is used during the decision making, this is not up to standard and it is not consistently used; as such most participants indicated that there are indeed situations where evidence is not used to inform decisions but rather personal opinion and experience dominate. Furthermore, participants lamented that it is difficult to access the evidence, in other cases insufficient evidence is available and also resistance to change 
the status quo and actively start utilizing evidence when assessing health technologies. For instance, some of the staff members in the Ministry of health were trained on evidence use and synthesis but their supervisors are not keen on utilizing evidence hence impeding proper health technology adoption process ending up using personal opinions.

"... we train them and then there's a mentorship program. We found that after one year most people were not using the skills which they gained from it... There were some other barriers which were aired out by the trainees but ... some of them were like ... the seniors don't even value evidence or something like that they can't access the evidence." (FGD Res.08)

Lack of a standardized framework for key considerations for health technology adoption: Most participants felt there is no distinct criteria for assessing medical devices and equipment. Despite having policies such as EHP, HSSP II which have spelt out to use when making decisions, these values have not been harmonized to facilitate their effective use in identifying, prioritizing and adopting different health technologies. The following are the core values: Primary Health Care, Human Rights-Based Approach and Equity, Gender Sensitivity, Ethical Considerations, Efficiency and Effectiveness, Transparency and Accountability. Coordination and collaboration, Community Participation, Evidence-based decision-making, Decentralization, Appropriate Technology, Universal Health Coverage (UHC), Demonstrable Value for money, Accountability for results and expenditures, and Sustainability of the health technologies. These values are used in isolation of each other and each TWG comes up with their values and norms to use when doing the health technology assessment, as highlighted by the quote below:

"The TWGs usually agree so for example, the EHP, the current EHP there was agreement, how are we going to say this intervention or technology makes it or not? The TWG agree on criteria and then jets interventions into those criteria. Adoption, however, adoption as far as I know is open ended because as... said these decisions come to SMT. Now, SMT doesn't have criteria in black and white to say 'if you meet this or you don't meet this then we'll do this or we won't do this." (FGD Res. 05)

Difficult to implement costeffective interventions: Participants from both FGD and KIls expressed that the procurements for the adoption of medical devices and medicines conflict with other policies like "Buy Malawi". Under "Buy Malawi", $60 \%$ of the procurements should be purchased locally even if cheaper options are elsewhere. As such while technical experts will identify cost-effective interventions, during adoption of such medical devices and medicines, PAM and Central Medical Stores Trust will procure most of the products from Malawian manufacturers which bloats prices hence defeating the implementation of cost-effective interventions.

Political interference. Key informants lamented that at times decision makers accept technologies from donors though they are not in line with the health technology assessment procedures because politicians are in favor of those technologies regardless of the effect the technology has on the health system. This inhibits the tenets of transparency and accountability due to the inadequate processes being followed.

Pressure from donors: The study also established that some technologies are adopted in Malawi not from need but because a prominent donor has suggested so. As a result, technologies which are difficult to sustain and maintain are adopted.

Lack of capacity at PMRA: It was also revealed that PMRA fails to do assessments on account of shortage of equipment and staff. As such they rely on information from WHO and other big regulatory authorities from Europe and USA to decide to adopt technologies, as one respondent reported, "Like the PMRA, they have that mandate to approve vaccines, ARVs and all those things. But sometimes they don't have the labs well equipped to assess the potency of a drug. They depend on the report from a manufacturer or other international similar regulatory authorities." (KII 05). This becomes problematic when the medicines are produced from countries or continents which do not have robust regulatory authorities and yet Malawi does not also have the capacity to do assessments. They end up sending the samples to other countries like Kenya which turns out to be costly.

Given the vast amount of knowledge and pertinent challenges that were highlighted by the study participants Table 2 is a SWOT analysis examining the strengths, weaknesses, opportunities and threats that are prevalent in the informal HTA mechanisms that exist in the Malawi health care system. The analysis is broken down through the streamlined hierarchical system of decision making that includes MOH SMT, TWG and PMRA.

Table 2: Strengths, Weaknesses, Opportunities and Threats of informal HTA Mechanisms 


$\begin{array}{ll}\text { Strengths } & \text { Greater alignment of policy question to health system goals } \\ \text {-Senior management composition could mitigate any risks in } \\ \text { decision making arising from weak evidence base including on } \\ \text { cost-effectiveness } \\ \text {-The multi-stakeholder composition of TWG members provides } \\ \text { scope for transparency and accountability. }\end{array}$
scope for transparency and accountability.
-Available capacity for handling issues of efficacy and effectiveness

-Has legal Mandate-Act of Parliament

•Has significant level of independence

-Is already funded by public, donor and private funding streams

- Its mandate already stretches across government, donor, and privately funded technologies

-Minimal political interference as oversight is through a Board of Trustees

-Has robust internal research capacity for pharmacovigilance

$\begin{array}{ll}\text { Weaknesses } & \cdot \text { SMT can be pressured by donors } \\ & \text { Political economy interests of top decision makers could prevail } \\ & \text { over value and evidenced-based decision making } \\ & \cdot \text { Weak institutional mechanism for evidence synthesis }\end{array}$

Opportunities $\quad \cdot$ It is an existing decision making structure of $\mathrm{MOH}$

-Any identified institutional and funding gaps can be reasonably addressed

-TWG structure is acceptable by donors and wider stakeholders

-TWG structure provides access to top researchers who can contribute to the evidence process at no cost to Government

$\begin{array}{ll}\text { Threats } & \cdot \text { Political interference } \\ & \cdot \text { Could be influenced by political economy considerations }\end{array}$

- Its statutory corporation status could limit scope for its execution in the event of interference by $\mathrm{MOH}$ and other central government institutions

- It does not have capacity in cost-effectiveness analysis

- Poor coordination with health technology procurement institutions

- Lack of WHO prequalification of the laboratory limits assessment of some pharmaceuticals

-New regulations extend its mandate to cover all health technologies rather than medicines only

• Has trust of all stakeholders in health technology

\section{Institutionalizing HTA in Malawi: demand and options for institutionalization}

For institutionalization of HTA, there must be demand for it. This was reviewed by asking FGD and KII informants whether or not HTA would improve decision making in the health sector. From this question, we further inquired what form HTA institutionalization could take. Based on interview results, the need for HTA was extensively highlighted, deemed necessary and needed. The participants expressed that an institutionalized HTA would fill a regulatory gap for HT adoption across multiple channels. The participants further iterated that an institutionalized HTA will facilitate cost-effectiveness considerations into decision-making for both medical devices and medicines including donations. A participant suggested that HTA "will serve as a guiding tool to make sure that there is competition on the market" and ensure objectivity when ... "selecting these manufacturers or suppliers" (KII_06). The participants further proffered that, the institutionalization of HTA would provide coordination among stakeholders involved and improve relationships across institutions. The participants thereby reported consensus that institutionalizing HTA in Malawi will improve decision-making regarding technology adoption, contribute to efficiency, equity and transparency in health financing, thereby leading to improved service delivery and results.

\section{Form and Scope of institutionalized HTA}

Based on the functions already undertaken by PMRA, most respondents who were conversant with HTA processes or practice suggested that efforts towards institutionalizing HTA should constitute empowering the PMRA. PMRA has a new regulatory framework that was drafted and has "expanded the scope to include all aspects of technology such as medical devices and traditional medicine currently excluded in the PMRA Act". (KII_06). Therefore, the respondents explained that PMRA could be a baseline pilot for the institutionalization of HTA in Malawi by capacitating it.

Other possible options for institutionalization was the suggestion that HTA be established as a standalone institution independent of MoH. A third option for institutionalization that the respondents considered was setting up an HTA body within an appropriate Academic institution established based upon the strong research culture that exists in academia upon which the evidence base for HTA could be harnessed. A fourth option suggested was establishing a unit specializing in HTA within a department of the MoH, possibly under the Public Health Institute of Malawi (PHIM). The respondents highlighted that this would ensure that HTA is "housed within the ministry as this is where the majority of the decisions are made in regards to health technology" (KII_08). A final option mentioned was to set up HTA as a "committee and eventually develop that into a full department within one of the government functions" (KII_08), once the value of HTA has been established.

\section{Funding for HTA in Malawi}


The participants expressed multiple ways in which an HTA could be funded in Malawi. These options included but were not limited to an HTA framework developed and implemented with funds from the government, international partners such as the WHO, UNICEF, GAVI, GIZ, USAID, and organizations like Partners in Health, CHAl, etc. Another proposition that was brought forth was to have a subscription-based system for all partners that would have a role in the HTA framework.

\section{Benchmarking HTA Institutions: WHO, UK, Thailand, Ghana, Ethiopia and South Africa}

The institutional contexts of HTA agencies are highly variable, presenting differences in the organization of the HTA process "chain of assessment" stages (prioritization, evaluation, appreciation, dissemination, and implementation of results/recommendations) (11). The aim of the literature review was to benchmark HTA structures in various countries in order to understand various HTA themes and values and how institutionalization is conducted in varying contexts $(12,13)$. The diversity of the organization of HTA in different countries with regard to mission, structure, financing, priority setting and impact on decision-making was assessed using examples of known HTA agencies such as the WHO guidelines for effective HTA institutionalization, the UK NICE structure, Thailand, Ghana, Ethiopia, South Africa and Switzerland.

WHO: The WHO has been a key player in endorsing HTA as critical tool into evaluation mechanisms. The WHO proffers that successful HTA programs require an appropriate education and training strategy targeted at expertise, organization and staff qualification. It is leveled that the varying sectors of health technology systems, organizations, processes, procedures, devices and drugs should be subject to assessment, comparison and continuous improvement (6). The WHO highlights generic areas such as, "a focus on clinical databases on low-cost common technologies versus high technology; correlation between output and content; bottom-up/top-down synergy; driven by professions not by industry; increase equity and access to emerging technology may be especially helpful in this regard" (6).

UK: As an independent institution, the National Institute for Health and Clinical Excellence (NICE) provides a roadmap in which quality health can be achieved in the United Kingdom (12). NICE is responsible for appraising technology directed at clinical and cost effectiveness of new or existing medicines and treatments and then providing corresponding feedback to the NHS (13). NICE has a major focus on technologies that are in the realm of pharmaceuticals, devices, diagnostics, procedures and health promotion mechanisms (14). The Key principles (normative values) of NICE guidance development are validating process that includes focused questions, stakeholder input, independent robust evidence assessment, independent advisory committee, multiple perspectives, transparent process \& decision making, genuine and public consultation and regular review (12).

Thailand: HTA institutionalization in Thailand has been viewed as a leading institutional structure globally that is conducted in a manner that incorporates evidence generation and use in policymaking, building capacity of HTA practitioners, organizations, system infrastructure, and collaborations (15). In Thailand, the HTA mechanisms is propagated into decisions of coverage and plays a prominent role in the conceptualization of the List of National Essential Medicine as well as considerations into UHC benefits package scheme (15). The guiding features of this method for institutionalization propagate values such as political will and leadership, capacity building on HTA-related disciplines, adequate resources, technical expertise, and data (6).

Ghana: The institutional arrangements for HTA in Ghana are in the preliminary stages. Currently HTA is built to initiate institutionalization to inform policy making, priority setting for health interventions, reimbursement, pricing of pharmaceuticals and other health technologies (16). HTA in Ghana was propagated by the Minister in the Ministry of Health with the inauguration of the HTA Steering Committee, Technical Working Group and Secretariat, who oversee the development of HTA under the auspices of the Ghana Ministry of Health (16). The Ghanaian approach to HTA is spelt out in the countries Ministry of Health's strategic plan, whose identified purpose is strengthening the science and practice of HTA in support of evidence-based decisions in order to advance health provision outcomes in their nation.

Ethiopia: Health technology assessment (HTA) in Ethiopia exists in a fragmented manner. This disjointedness, called for an in-depth review of the application of HTA in the context in line with the populations health needs. Among the institutional capacity, the Health Economics and Financing Analysis (HEFA) team was established within the Finance Resource Mobilization Department under the Ministry of Health (17). The HEFA mandate champions the application of evidence-based health care decision making in Ethiopia by organizing available evidence, costing interventions, and defining effectiveness measures of the different health programs and then supporting policymakers at the national and regional levels (17).

South Africa: Health care in South African aims to ensure that the most prominent and basic health needs of the population are met (18). Thus HTA was adopted as mechanism to anchor the ideals of UHC. HTA in South Africa is both a technical and political process, involving a range of stakeholders, systems, disciplines and viewpoints (10). The HTA processes in South Africa seeks to be effectively anchored on the views and experiences of a broad range of stakeholders, across income quintiles and sectors, and seeks to develop a sense of ownership (10).

\section{Discussion}

This section presents a discussion on the findings of the study and recommendations of plausible ways in which HTA can be institutionalized in a low income setting such as Malawi given the pertinent challenges that exists in its health sector. After a thorough understanding of the dimensions that guide decision making in health technology prioritization and adoption in Malawi, the need for an institutionalized HTA mechanism was found to be acceptable and implementation deemed feasible. In a lower resource setting such as Malawi, HTA has a potential to play a significant role in the incorporation and utilization of health technology processes, by contributing to the equitable provision and access to healthcare services, more efficiency in the allocation of resources, better effectiveness and quality of services, and stronger financial sustainability of the healthcare system (11).

\section{The Nature of Decision Making and HTA in Malawi}


The study found that, there is no institutionalized HTA mechanism in Malawi though some partial HTA processes exist at different points in the health technology adoption process. The partial HTA mechanisms exist primarily in the MoH Senior Management Team, Technical Working Groups, and the PMRA that were all found to have varied roles of effectiveness. It was established that Malawi relies primarily on committee based decision making processes that do not utilize consistent methods across channels also making them prone to difficulty in implementing cost-effective interventions, political interference, pressure from donors, limited and unsystematic use of evidence, partial adequacy of capacity at PMRA. These findings are seemingly consistent with the status of HTA in other low income countries were either HTA does not exist at all, or exist as partial processes at different phases of the health technology adoption or prioritization process (3).

Network et al (2018) notes that, even though a formal HTA program might not be in place in a given country, decision-making about the adoption of new technologies may be part of the operational routine of health authorities and health service providers. In this case, decision making is frequently based on unilateral industry information, particular interests of individuals or 'gut feelings' which may prove futile, unreliable and even disruptive to the decision making process (24). This is found evident in the case of Malawi were decision making on health technology prioritization and adoption is marred by limited or inadequate evidence use, political interference, value misalignment between the Essential Health Package (EHP) and other corresponding policies such as the Essential Medicines List and The Standard Treatment Guidelines, The National Health Policy and so forth. To rectify such challenges, (24) proffers that health systems shift from "sporadic decision making" to decision-making processes that follow systematic modern principles such as Evidence Based Medicine (EBM), cost effectiveness and patient centered services that have the capacity to translate into the establishment of an institutionalized HTA.

\section{Institutionalizing HTA: Moving from Informal Assessments to Formal, Harmonized and Institutionalized HTA}

In general, HTA has its prominence in high and middle income countries. Major focus in the world has been placed on England and Wales that are deemed a pioneer institution were the National Institute for Health and Clinical Excellence conducts appraisals using the evidence coming from HTA, in a process that leads to guidance (25); a term they refer to as HTA beyond decision making (5). Similarly, in middle - high income countries such as Thailand, HTA has been formally integrated into coverage decisions, including in the development of the National List of Essential Medicines and the Universal Health Coverage Scheme benefits package (26). Drawing lessons from NICE and HITAP and how they are expanding their mandate on HTA to other low income countries such as Myanmar, The Philippines, Vietnam and Columbia could also provide firm direction on a potential pathway Malawi can take in institutionalizing HTA (5).

Literature shows that moving to a formalized and systematic HTA program requires a solid commitment from governmental authorities and a designated and motivated team of professionals that take charge of the HTA development plan. (10) reports that there is no 'one-size-fits-all' for delivery of HTA, rather HTA can be institutionalized in varying degrees according to each relative context. A good approach to HTA institutionalization can be to design a system that caters to the unique policy needs of that context, becoming an institution that works with inbred funding structures and boosts the nature and availability of evidence and existing approaches to decision-making (18). And whether the HTA is successful or not can be established as the extent to which it contributes to defined policy objectives such as achieving value for money and improving health outcomes, and addressing inequalities and access to health technologies (10).

\section{Institutionalizing HTA in an LIC: The Case of Malawi}

To establish a formalized HTA institution in an LIC such as Malawi there is need to establish a mandate among preliminary stakeholders that would warrant a critical role in the establishment of the institution. These stakeholders would be the primary parties responsible for decision making (policy brokers) and relevant parties involved in the prioritization and adoption of health technologies in the country. In Malawi, these stakeholders could potentially be the MoH, Central as well as District Hospitals, Civil Society, CMST, Development/ Donor Partners, CHAM, PHIM and regulatory authorities such as the Medical Council and PMRA. Secondly, there would be a need for the development of a legal framework, establishment of institutional arrangements, definition of procedural aspects of assessment and appraisal and a proposed monitoring and evaluation component of the HTA mechanism (27). Importantly, some reforms would be required such as having the Planning and Policy Directorate of the Ministry of Health decide whether to continue with current practice of committee based decision making processes or grow towards a hybrid, or a formal institutionalized HTA.

In the case of Malawi, given the partial HTA processes that exist, a major premise of institutionalizing HTA could boarder on growing from or expanding on prioritization an adoption processes (decision making structures) that currently exist within relevant institutions explored within the study. These institutions could be fine-tuned as a baseline for building a comprehensive HTA institution with distinct as well as complementary steps and processes that aid cost effective decision making. These processes could be, (a) developing HTA capacity of PMRA to act as an HTA agency operating under regulation, (b) Strengthening capacity of MoH Senior Management and District Councils in HTA agenda setting as a phased approach towards institutionalizing HTA,

(c) Establishing Cost-Effectiveness Analysis capacity of MoH and TWGs to aid evidence generation and synthesis, (d) Developing capacity to conduct cost - effectiveness analysis and evidence generation within academia, as well as (e) building sustainable financing for HTA.

\section{Developing HTA capacity of PMRA - HTA Agency}

As found in this study, the PMRA mandate for Malawi gives regulatory oversight of registration of health technologies but not the authority over what technologies to fund and not to fund. To take on board the function of a fully functional HTA institution. PMRA could take up the role of a national 'HTA Agency' as a considered alternative for HTA. PMRA could be tasked as HTA Agency with a governmental mandate fulfilling the role of a policy body. commissions specific HTA reports and functions as a clearing house between academia and the 'end-users' (health ministries, private health providers, health insurances, etc) (24). Since PMRA already has a board and is regulated by a Parliamentary Act, this could provide significant independence of the 
HTA division within PMRA. An additional advantage of developing capacity with PMRA is that it would allow incorporating cost-effectiveness analysis studies in pharmacovigilance studies, a key role that PMRA plays in post technology adoption decision. Regarding the actual capacity that could be institutionalized, institutional assessment would be needed to establish the existing capacity, the gaps and feasible structure given mandate and funding arrangements.

\section{Strengthening Capacity of MOH Senior Management and District Councils in HTA agenda setting}

With HTA capacity developed within PMRA, there would be a need to strengthen the HTA agenda setting role of MOH and district councils since they are the fund holders of Government money. This capacity building could extend to developing competencies of these institutions as gate-keepers of donor procured or donated technologies so that principles of cost-effectiveness are also extended to products produced on behalf of Government facilities outside of Government arrangements. This would also require strengthening the use of PMRA HTA institutional processes on both Government and donor funded technologies as well donations in kind. This will require undertaking a needs assessment and developing responsive capacity in line with the overall HTA architecture. This will then inform both the national level institutional setup consistent with optimal HTA decision making.

\section{Establishing Cost-Effectiveness Analysis capacity of MOH and TWGs}

Within $\mathrm{MOH}$ institutional arrangements, there would be a need to develop internal capacity to support the agenda setting and funding decision processes by strengthening the capacity of the newly established Health Financing Division (HFD). Presently these functions are diffused between the budget division and the policy development division. The mandate of the HFD is to identify and facilitate cost-effectiveness assessments of both government and donor funded interventions including technologies. Also, the HFD is the custodian of Memorandums of Understanding (MOUs) between the Government and its development partners in the health sector. This places the HFD as an ideal link between HTA and implementation scale up of MOH and donor funded technologies. Also, at the core of the health financing reforms being pursued, value for money and sound public financial management are the pillar stones through which $\mathrm{MOH}$ aims to mobilize fresh health financing for UHC. Therefore, HTA capacity within the HFD could contribute to these efforts. The health financing TWG, to which the HFD is the secretariat, provides a technical advisory role to the MOH Senior management. This could provide a layer between HTA evidence demand and $\mathrm{MOH}$ decision making. This suggests that for HTA, going forward, capacity building of the HFD and TWG is key for institutionalization.

\section{Developing capacity to conduct Cost Effectiveness Analysis and Evidence Generation within Academia}

Also, the Government facilitated and funded the Health Economics and Policy Unit (HEPU) at the Kamuzu University of Health Sciences. This health policy analysis capacity building collaboration could be tasked among others with the policy analysis function in support of the HTA capacity gaps within the $\mathrm{MOH}$ and PMRA. This specialized capacity within HEPU can be developed either as "offshore" HTA capacity fully aligned to MOH and executing HTA evidence and knowledge management function in a sub-contractor capacity. This option will allow MOH to focus on agenda setting and decision making in line with its policy oversight mandate while HEPU and other academic institutions with relevant HTA capacity can provide the evidence support. At the time of this study, the Government, through the Health Service Joint Fund, had already approved a grant to support evidence generation in health economics, equity and ethics in support of $\mathrm{MOH}$ and donor joint decision making. One deliverable of the grant is to support $\mathrm{HTA}$ institutionalization. Over time, this deliverable could be strengthened and institutionalized as a robust HTA evidence support structure.

\section{Building sustainable Financing for HTA}

HTA bodies may be eligible to receive public funding and be established by ministries of health (at national, provincial or regional level), receive a mixture of private and public funding, be independent of governments, be situated within academia or initiated by organizations of health professionals (28). For effective HTA, sustainable and ear-marked funding for the HTA mechanisms with PMR, HFD and HEPU should be identified, explored and sustained over time. For academic institutions, this could be supplemented by research grants that support cost-effective analysis. Finally, funding for submissions for privately funded requests from companies through the manufacturers. Sufficient investment funds should be made available to train professionals in HTA work. Funding for the recurrent operational costs of the established HTA structure should be identified and secured on a long term basis. HTA work is no longer done in national isolation. The national HTA concept should include an international network strategy right from the beginning (24).

\section{Conclusion}

HTA in LMIC countries such as Malawi is feasible and necessary to promote equitable, efficient, fair and transparent resource allocation and prioritization. The study discovered that there are three types of structures that can exist in decision making structures; committee based systems, a hybrid of committee based and partial HTA institutional mechanisms and a fully functional institutionalized HTA. Malawi shows a strong demand and need for an institutionalized HTA. The study suggests that any HTA institutionalization in the short to medium term should focus on strengthening the capacity of the PMRA to appraise CEAs. The institutionalization efforts should also concurrently develop MOH HTA capacity by strengthening the commissioning role of SMT and advisory role of the HF TWG. This should be through the newly established health financing division which is already secretariat to the HF TWG. It should further develop capacity to conduct CEAs within academia and $\mathrm{MOH}$ health financing division. Overall, the evidence reveals that rather than creating new HTA institutions explicitly, country specific assessments should precede with the development of value frameworks that aid the decision making processes in adoption but also concurrently aid conflict of interest management from political pressures. These can be the specific recommendations on the basis of which a national level institutionalization framework could then be developed and implemented.

\section{Abbreviations}


$\mathrm{CDC}=$ Centre of Disease and Control

CMST $=$ Central Medical Stores Trust

$\mathrm{CHAI}=$ Clinton Health Access Initiative

CHAM $=$ Christian Health Association of Malawi

COMREC $=$ College of Medicine Research Committee

CEA $=$ Cost Effectiveness Analysis

DHTSS $=$ Department of Health Technical Support Services

EHP = Essential Health Package

FSIOS = Federal Social Insurance Office Switzerland

FGD = Focus Group Discussion

HEPU $=$ Health Economics and Policy Unit

HEFA $=$ Health Economics and Financing Analysis

$\mathrm{HF}=$ Health Financing

HSSP II = Health Service Strategic Plan II

HSJF = Health Services Joint Fund

HTA = Health Technology Assessment

HFD = Health Financing Division

HITAP = Health Intervention and Technology Assessment Program

INAHTA = International Network of Agencies for HTA

IPC = Internal Procurement Committee

KuHes = Kamuzu University of Health Sciences

$\mathrm{KII}=$ Key Informant Interviews

LICs = Low-income countries

$\mathrm{MOU}=$ Memorandum of Understanding

$\mathrm{MOH}=$ Ministry of Health

NICE $=$ National Institute of Clinical Excellence

PMRA = Pharmacy and Medicines Regulatory Authority

PAM $=$ Physical Asset Management

PIU = Project Implementation Unit

PHIM = Public Health Institute of Malawi

SMT = Senior Management Team SMT

SNHTA = Swiss Network on HTA

TWG $=$ Technical Working Group

$\mathrm{UHC}=$ Universal Health Coverage

UNICEF = United Nations International Children's Emergency Fund

WHO $=$ World Health Organization 


\section{Declarations}

\section{Availability of data and materials}

The datasets used and/or analyzed during the current study are available from the corresponding author on reasonable request.

\section{Authors' contributions}

JMB conceptualized the study and supervised the writing. FJ led the manuscript writing, FKC conducted data management, analysis and supported the writing up of the results section; NN supported literature review, DN supported the writing up of the introduction and discussion; IKC and DN advised on the data analysis; GM and PT advised on policy insights on the paper; MD provided policy insights on the paper; WN reviewed the manuscript, MJK reviewed the manuscript, TC reviewed the paper and provided concept advise, JC reviewed the manuscript and provided policy insights; MC reviewed the paper and provided concept refinement; PR and MS contributed to the conceptualization of the study. All authors read and approved the final manuscript.

\section{Acknowledgements}

The authors would like to thank Michael Drummond and Francesco for contributing to the conceptualization of the HTA Framework. The authors would also like to thank extend their immense gratitude to all HEPU members on their continued support throughout the data collection and analysis and drafting of the manuscript. The authors bid gratitude to all the participants in their respective positions for data collected during the study.

\section{Funding Sources}

UKRI through Centre for Health Economics at the University of York under the Thanzi La Onse Project.

\section{Availability of data and materials}

The datasets used and/or analyzed during the current study are available from the corresponding author on reasonable request.

\section{Ethics approval and consent to participate}

The study observed strict ethical considerations approved by the College of Medicine Research Committee (COMREC) in Blantyre, Malawi (protocol \#: P.10/19/2820). All methods were performed in accordance with the relevant guidelines and regulations (declaration of Helsinki). Verbal and written consent was obtained from all the participants involved; a copy of the Consent Form used has been included in the supplementary materials. Each study participant consented to participate in the study before any study activities. The purpose of the study was explained to all participants who were approached. Participants who took part in the FGD were requested to observe confidentiality. To protect the confidentiality of the participants' information; no identifying information was included on the interview transcripts or FGD. The audios and transcripts were kept in a password protected computer with access limited to the researchers.

\section{Consent for Publication}

All participants and authors consented to the publication of the study.

\section{Competing interests}

The authors declare they have no competing interests.

\section{Author Details}

Joseph Mfutso-Bengo ${ }^{1,2^{*}}$ Faless Jeremiah ${ }^{1}$, Florence Kasende-Chinguwo ${ }^{1}$, Wingston $\mathrm{Ng}^{\prime}$ ambi $^{1}{ }^{1,3}$, Nthanda Nkungula ${ }^{1}$, Isabel Kazanga-Chiumia ${ }^{1}$, Mercy Juma-Kunyenga ${ }^{1}$, Marlen Chawani ${ }^{1,6}$, Jobiba Chinkhumba ${ }^{1}$, Pakwanja Twea ${ }^{5}$, Gerald Manthalu ${ }^{5}$, Dominic Nkhoma ${ }^{1}$, Tim Colbourn ${ }^{7}$ Paul Revill ${ }^{4}$, Mark Sculpher $^{4}$ Michael Drummond ${ }^{4}$

\section{References}

1. Tantivess S, Chalkidou K, Tritasavit N, Teerawattananon Y. Health Technology Assessment capacity development in low-and middle-income countries: Experiences from the international units of HITAP and NICE. F1000Research. 2017;6(December 2017).

2. Prinja S, Jyani G, Gupta N, Rajsekar K. Adapting health technology assessment for drugs, medical devices, and health programs: Methodological considerations from the Indian experience. Expert Rev Pharmacoecon Outcomes Res. 2021;

3. Kriza C, Hanass-Hancock J, Odame EA, Deghaye N, Aman R, Wahlster P, et al. A systematic review of health technology assessment tools in subSaharan Africa: Methodological issues and implications. Heal Res Policy Syst. 2014;12(1).

4. O'Rourke B, Oortwijn W, Schuller T. The new definition of health technology assessment: A milestone in international collaboration. Int J Technol Assess Health Care. 2020;36(3):187-90. 
5. Tantivess S, Chalkidou K. Health Technology Assessment capacity development in low- and middle- income countries: Experiences from the international units of HITAP and NICE Health Technology Assessment capacity development in low- and middle-income countries: Experiences from th. 2017;(January 2018).

6. WHO Regional Office for Europe. Institutionalization of Health Technology Assessment Report on a WHO Meeting. 2001;(July 2000):1-24. Available from: http://www.euro.who.int/_data/assets/pdf_file/0016/120247/E72364.pdf

7. Chalkidou K, Glassman A, Marten R, Vega J, Teerawattananon Y, Tritasavit N, et al. Priority-setting for achieving universal health coverage. Bull World Health Organ. 2016;94(6):462-7.

8. Culyer AJ. Cost-effectiveness thresholds in health care: A bookshelf guide to their meaning and use. Heal Econ Policy Law. 2016;11(4):415-32.

9. Tantivess S, Chalkidou K, Tritasavit N, Teerawattananon Y. Health Technology Assessment capacity development in low- and middle-income countries: Experiences from the international units of HITAP and NICE. F1000Research. 2017;6(January 2018).

10. Siegfried N, Wilkinson T, Hofman K. Where from and where to for health technology assessment in South Africa? A legal and policy landscape analysis. South African Heal Rev 2017 [Internet]. 2017;41-8. Available from: http://www.

11. Novaes HMD, Soárez PC de. Health technology assessment (HTA) organizations: dimensions of the institutional and political framework. Cad Saude Publica. 2016;32(suppl 2):1-14.

12. Garner S, Adviser T. HTA as input to NICE guidance in England and Wales What is NICE?

13. Craig AM. Understanding health technology assessment. Med Device Technol. 2000;11(7):32-7.

14. DJ W. National Institute for Health and Care. Annu Meet Am Soc Clin Psychopharmacol [Internet]. 2019;(April):1-104. Available from: https://www.nice.org.uk/guidance/GID-NG10013/documents/draft-guideline

15. Leelahavarong P, Doungthipsirikul S, Kumluang S, Poonchai A, Kittiratchakool N, Chinnacom D, et al. Health Technology Assessment in Thailand: Institutionalization and Contribution to Healthcare Decision Making: Review of Literature. Int J Technol Assess Health Care. 2019;35(6):467-73.

16. Peacocke E, Asare B, Ackon A, Espeland AL, Chola L, Dolphyne A, et al. Summary report of published literature on capacity building for skills related to health technology assessment in Ghana. (March 2021):1-36.

17. Inotai A, Pékli M, Jóna G, Nagy O, Remák E, Kaló Z. Attempt to increase the transparency of fourth hurdle implementation in Central-Eastern European middle income countries: Publication of the critical appraisal methodology. BMC Health Serv Res. 2012;12(1).

18. Inform TO, Selection THE, Medicines OF, The TO, African S, Essential N, et al. Health Technology Assessment Methods Guide To Inform the Selection of Medicines To the South African. 2021;(June).

19. Charles J, Scranton J, Wright J. Essential Package of Health Services Country Snapshot: The Republic of South Sudan. 2015;(July). Available from: https://www.hfgproject.org/essential-package-of-health-services-country-snapshot-the-republic-of-south-sudan/

20. Government of Malawi. Health Sector Strategic Plan II (2017-2022): Towards universal Health coverage. 2017;122. Available from:

https://extranet.who.int/countryplanningcycles/sites/default/files/planning_cycle_repository/malawi/health_sector_strategic_plan_ii_030417_smt_dps.pdf

21. MoH. Malawi Standard Treatment Guidelines (MSTG ) 5 th Edition 2015 Guidelines ( MSTG ). 2015;687.

22. Butao, Doris, Barbara Felling and PM. laboratory services and supply chain assesment in Malawi, Task Order 1 . Arlington, Va USAID I Deliv Proj Task Order 1. 2009;(accessed on August, 2021):1-104.

23. August P, Arthur P, Mutharika P. Gazette Extraordinary. Int Leg Mater. 1968;7(3):626-32.

24. Network E, Health FOR, Assessment T. Handbook on Hta Capacity Building. Health Technol Assess (Rockv). 2008;(October).

25. Campbell B, Knox P. Promise and plausibility: Health technology adoption decisions with limited evidence. Int J Technol Assess Health Care.

2016;32(3):122-5.

26. Williams K, Sassler S, Frech A, Addo F, Cooksey E. Health technology assessment. An introduction to objectives, role of evidence, and structure in Europe. J Health Soc Behav [Internet]. 2013;54:277. Available from: http://www.ncbi.nlm.nih.gov/pubmed/24055071

27. Edejer TT. Institutionalizing health technology assessment mechanisms: a how to guide. 2021.

28. Mueller DB, Tivey D, Croce D. Health-technology assessment: Its role in strengthening health systems in developing countries. Strengthen Heal Syst [Internet]. 2017;2(1):6-11. Available from: http://www.shsjournal.org/index.php/shsj/article/viewFile/45/22 
Figures

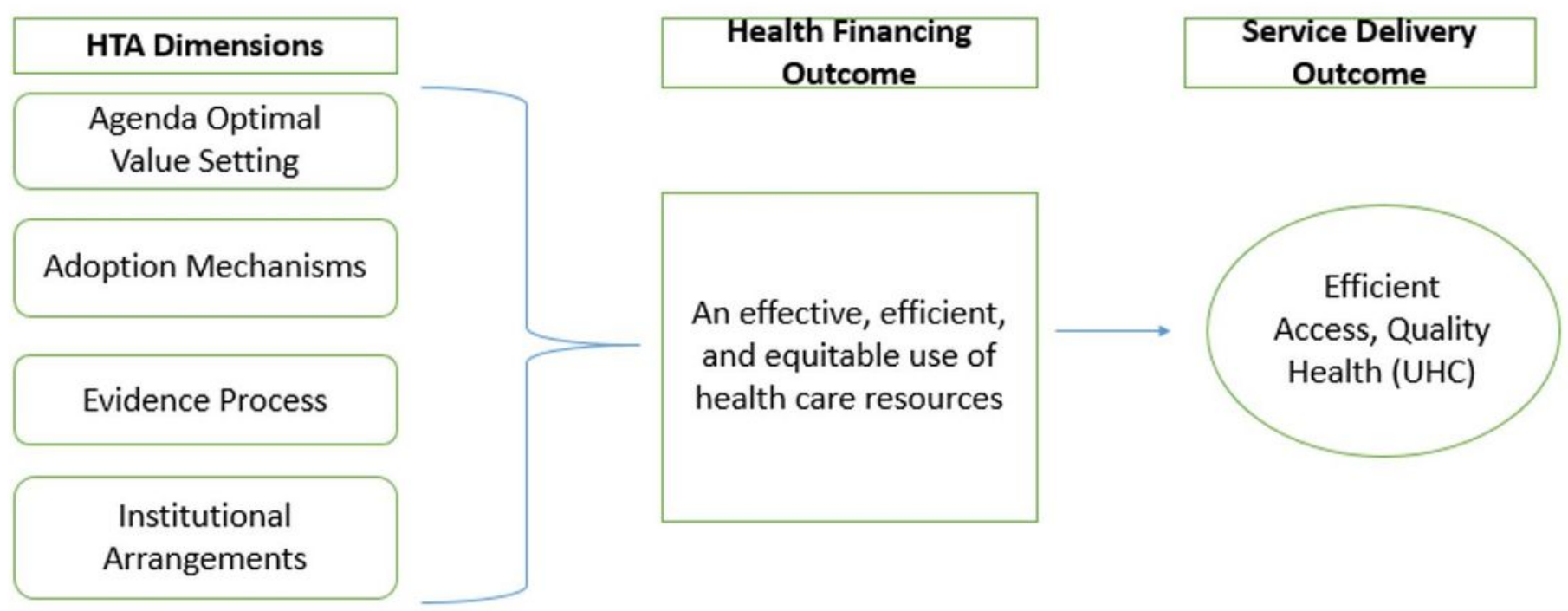

Source: Authors

Figure 1

Conceptual Framework for HTA Institutionalization

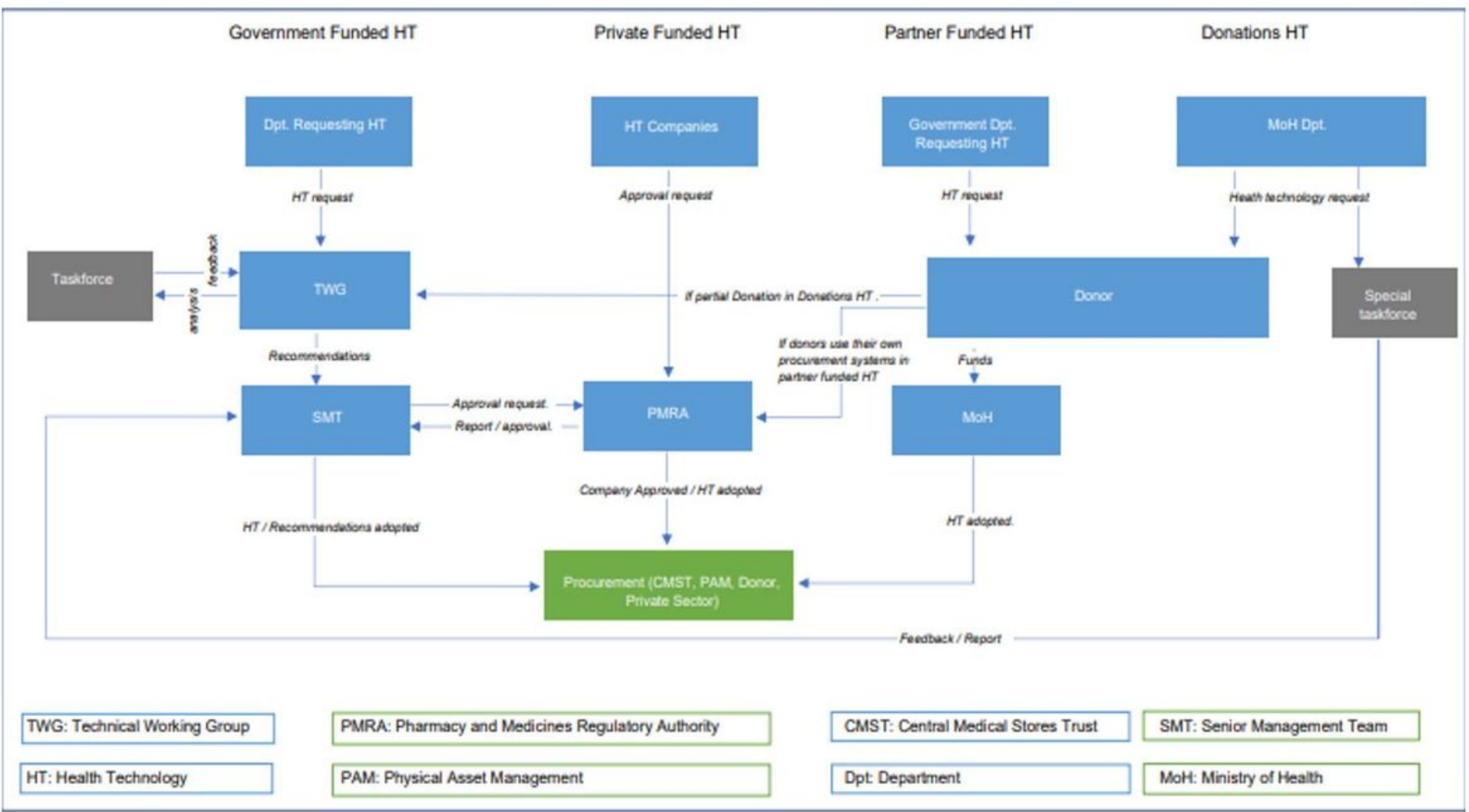

Figure 2

HTA Process for Pharmaceuticals and medical equipment

\section{Supplementary Files}


This is a list of supplementary files associated with this preprint. Click to download.

- HTAFocusGroupDiscussionGuideBMCSupplementary.docx

- HTAKeyInformantInterviewGuideBMCSupplementary.docx 